\title{
Optimal Replenishment Policy for Ameliorating Item with Shortages under Inflation and Time Value of Money using Genetic Algorithm
}

\author{
S.R. Singh \\ Department of Mathematics \\ D.N. College, Meerut (U.P.) India
}

\author{
Tarun Kumar \\ Department of Computer Science \\ Banasthali University, Banasthali \\ (Rajasthan), India
}

\author{
C. B. Gupta \\ Department of Mathematics \\ Birla Institute of Technology and \\ Science, Pilani (Rajasthan), India
}

\begin{abstract}
The implementation of supply chain has to reduce the total cost of system, but generally each component of a supply chain tries to find the best policy for its company and consequently tries to find a local optimum. Knowing that the sum of local optimum cannot constitute the global optimum, it is necessary to consider all costs of system simultaneously to find the optimal replenishment policy for all the components of a supply chain. Demand rate of the item is assumed to be a function of time known as ramp type function. Shortages are permitted and partially back-ordered. The back-ordering fraction is taken to be decreasing function of waiting time. We consider inflation and apply discounted cash flow in the problem analysis. Total cost of the system is formulated and optimal replenishment policy is derived, keeping in view the above factors of the system. We use Genetic Algorithm (GA) to solve the models. A numerical example and sensitivity analysis is shown to illustrate the models.
\end{abstract}

\section{Keywords}

Replenishment policy, ramp type demand, amelioration items , Optimization, Genetic algorithm

\section{INTRODUCTION}

The classical economic production quantity (EPQ) model assumes constant demand and infinite lifetime of items in inventory. Subsequent research efforts have led to the removal of these two restrictions in consideration of time varying demand functions and finite lifetimes for inventoried items. This is in line with common experience in day-to-day production and inventory management. The time-varying demand functions considered in most EPQ models are unidirectional, i.e. they are either continuously nondecreasing or continuously non-increasing function of time. The main objective of an inventory model is to calculate how much to replenish and when to replenish. After reviewing many inventory models of the past, it has been observed that many researchers included different factors to basic inventory model in order to study different environments/scenarios. One of the basic requirements to develop any replenishment policy for inventory system is its demand. The concept of both deterministic and probabilistic types of demand was discussed in much earlier phase of development of this study. In case of deterministic demand, constant demand was in vogue in earlier days. Since there are different inventory systems in real life, therefore researchers have had to choose demand patterns accordingly. One of the most common demand patterns that is suitable for almost all types of inventory systems is time varying demand pattern and a lot of work has been done in this direction till now. In order to represent the sales in different phases of the product life cycle in market, time dependent demand is suitable, e.g. the demand for inventory items increases over the time in the growth phase and decreases in decline phase. Silver and Meal (1969), Datta and Pal(1991), Chakrabarty et al. (1997) are few examples who considered linearly time dependent demand. Goyal(1987), Hwang (1995), Hwang(1997), Skouri and Papachristos (2003), Moon et al. (2005), Lin and Lin (2006) considered demand as continuous function of time. Benkherhouf and Balki (1997), Zhou, Lang and Yang(2004) considered demand as continuously increasingly function of time. Datta and $\mathrm{Pal}(1988)$ used power pattern in time dependent demand. As far as concerned two types of time dependent demand can be frequently found in papers: 1) linearly positive/negative trend in demand and 2) exponentially increasing/decreasing demand rate. This concept results in continuously increase/decrease in demand over time which is not realistic. Demand may increase during certain time periods and becomes constant after that. This type of demand can be represented by ramp type function. Hill (1995), Mondal and $\mathrm{Pal}(1998), \mathrm{Wu}$ and Quyang (2000), Panda et al. (2008), Skouri et al. (2009) considered ramp type time demand pattern. Besides demand, there are other important factors which affect an inventory model. Shortages are one of them. Any inventory model which is developed with no shortage can be redeveloped taking shortage into consideration. In above cited papers, the concept of shortages is excluded in some while included in others. When shortages arise, question of backordering arise simultaneously. Again two types of backordering can be experienced in inventory models, namely full backordering and partial backordering. Models considered by Goyal (1987), Datta and Pal (1991), Hariga (1995,1997), Chakrabarty et al. (1997), Wu and Quyang (2000), Moon et al. (2005) are some of the examples in which shortages are fully backordered. When customers have to face shortages, their response is different according the type of commodity and market environment. For a competitive market partial backordering plays a practical role. Wu (2001), Skouri and Papachristos (2003), Yang (2004), Lin and Lin (2006), Skouri et al. (2009) are few of researchers who considered shortages as partially backordered.

The replenishment problem has been traditionally treated from a multi-echelon and multi-product perspective (JenMing and Tsung-Hui 2005). A multi-echelon replenishment problem focuses on channel coordination issues for inventory replenishment, between upstream and downstream components of a supply chain, with the objective of minimizing total system costs (Sila et al. 2005). Moreover, multi-product replenishment problems aim to coordinate the replenishment of various items in the same family or same category in order to reduce the frequency of major setups and 
the related costs. This can be obtained by choosing an appropriate common replenishment frequency and lot-sizes within the family of items (Bahloul et al. 2008). Several previous works have studied the problem of multi-echelon, multi-product Supply Chain. Chen et al. (Cheng-Liang et al. 2004) have studied a multi-item inventory and transport problem with joint setup costs, referred to a joint replenishment problem.

\section{ASSUMPTIONS AND NOTATIONS}

The following assumptions and notations are used in formulating the models:

\subsection{Assumptions}

1. The inventory system involves single item.

2. Demand rate is depending on time given by

$$
D(t)=\left\{\begin{array}{l}
f(t), t<\mu \\
f(\mu), t \geq \mu
\end{array}\right.
$$

where $\mathrm{f}(\mathrm{t})$ is a positive continuous function of $\mathrm{t} \square$ $[0, \mathrm{~T}]$.

The function defined above is known as ramp type function.

3. Shortages are permitted and backordered at a rate $\mathrm{B}(\mathrm{t})$, which is a non-increasing function of $\mathrm{t}$ with $0 \leq$ $B(t) \leq 1$, where $t$ is the waiting time up to next replenishment.

4. Replenishment rate is infinite.

5. Deterioration and amelioration occur at constant rate

6. The deterioration and amelioration occur when the item is effectively in stock.

7. Inflation and money-value is considered.

\subsection{Notations:}

$\mathrm{D}(\mathrm{t}):$ Demand rate

$\mu \quad$ : Parameter for ramp type demand function $\theta(t)$ : Deterioration rate which is defined in terms of two parameter Weibull distribution as $\theta(\mathrm{t})=\alpha \beta \mathrm{t}^{\beta-1}$

$\alpha$ : Scale parameter of deterioration rate

$\beta \quad$ : Shape parameter of deterioration rate

$\gamma(\mathrm{t})$ : Amelioration rate which is defined in terms of two parameter Weibull distribution as $\gamma(\mathrm{t})=\mathrm{ght}^{\mathrm{h}-1}$

g : Scale parameter of amelioration rate

$\mathrm{h}$ : Shape parameter of amelioration rate

$\mathrm{r} \quad$ : Constant representing the difference between discount rate and inflation rate

$\mathrm{c}_{\mathrm{p}}$ : Purchase cost per unit

$c_{o} \quad$ : Ordering cost per order

$\mathrm{c}_{\mathrm{h}} \quad$ : Holding cost per unit per unit time

$c_{a}$ : Amelioration cost per unit

$\mathrm{c}_{\mathrm{d}}$ : Deterioration cost per unit

$\mathrm{c}_{\mathrm{s}}$ : Shortage cost per unit per unit time

$c_{1} \quad$ : Lost sale cost per unit

W : Maximum inventory level at initial point

$\mathrm{I}_{\mathrm{i}}(\mathrm{t})$ : Inventory level at any time $\mathrm{t}$ for $\mathrm{i}^{\text {th }}$ phase, where $\mathrm{i}=1,2,3,4$

$\mathrm{t}_{1} \quad$ : Time up to which inventory ameliorates.

$t_{2} \quad$ : Time at which inventory level becomes zero

$\mathrm{T}$ : Time for one replenishment cycle

$\mathrm{B}(\tau)$ : Fraction of demand backordered, which is decreasing function of waiting time, given by $1 / 1+\delta(\tau), 0<\delta<1$

\section{MODEL FORMULATION:}

Replenishment is made at the beginning of the cycle i.e., at $\mathrm{t}=0$, which brings the inventory level maximum equals to $\mathrm{W}$. Amelioration/deterioration occurs as soon as item is received into inventory. Due to amelioration inventory accumulates till $\mathrm{t}=\mathrm{t}_{1}$. Due to demand and deterioration inventory level starts declining and reaches to zero at $\mathrm{t}=\mathrm{t}_{2}$. Shortage occurs during the interval $\left[t_{2}, T\right]$, which is partially backordered. Since the demand function is divided into two intervals, therefore three cases can be considered in order to represent the syste

\section{Case1: when $t_{1}>\mu$}

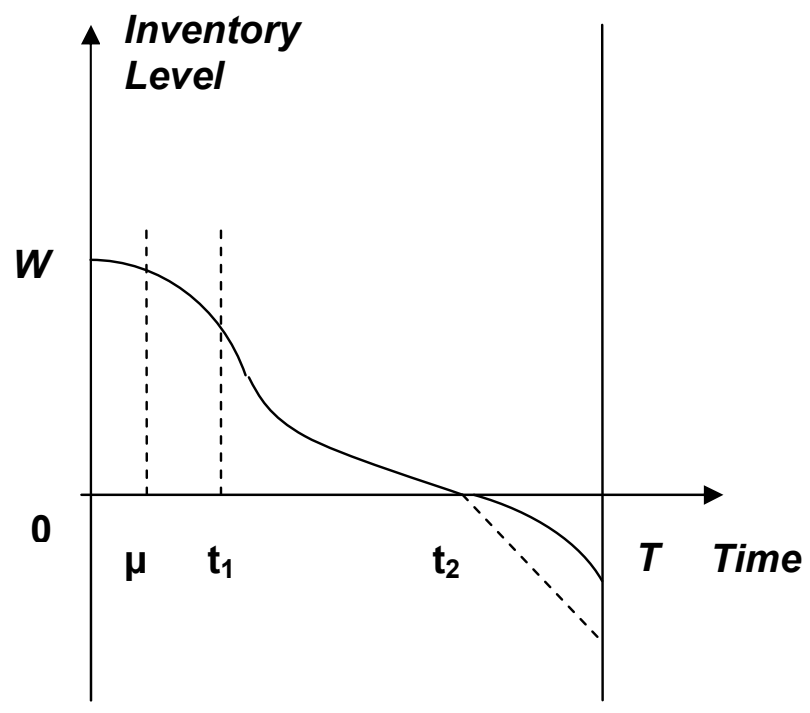

Fig. 1 : Variation of inventory level with time for Case 1. 
Inventory system can be represented by the following differential equations:

$$
\begin{array}{ll}
\frac{d I_{1}(t)}{d t}+\left(\alpha \beta t^{\beta-1}-g h t^{h-1}\right) I_{1}(t)=-(a+b t) & 0 \leq t \leq \mu \\
\text { with } I_{1}(0)=W & \\
\frac{d I_{2}(t)}{d t}+\left(\alpha \beta t^{\beta-1}-g h t^{h-1}\right) I_{2}(t)=-(a+b \mu) & \mu \leq t \leq t_{1} \\
\text { with } I_{2}(\mu)=I_{1}(\mu) & t_{1} \leq t \leq t_{2} \\
\frac{d I_{3}(t)}{d t}+\alpha \beta t^{\beta-1} I_{3}(t)=-(a+b \mu) & \\
\text { with } I_{3}\left(t_{2}\right)=0 & \\
\frac{d I_{4}(t)}{d t}=-\frac{(a+b \mu)}{1+\delta(T-t)} & t_{2} \leq t \leq T \\
\text { with } I_{4}\left(t_{2}\right)=0 &
\end{array}
$$

The solution of the above equations can be given as:

$$
\begin{aligned}
& I_{1}(t)=e^{-\alpha t^{\beta}+g t^{h}}\left[-a\left(t+\frac{\alpha t^{\beta+1}}{\beta+1}-\frac{g t^{h+1}}{h+1}\right)-b\left(\frac{t^{2}}{2}+\frac{\alpha t^{\beta+2}}{\beta+2}-\frac{g t^{h+2}}{h+2}\right)+W\right] \quad 0 \leq t \leq \mu \\
& I_{2}(t)=e^{-\alpha t^{\beta}+g t^{h}}\left[-(a+b \mu)\left(t+\frac{\alpha t^{\beta+1}}{\beta+1}-\frac{g t^{h+1}}{h+1}\right)+b\left(\frac{\mu^{2}}{2}+\frac{\alpha \mu^{\beta+2}}{(\beta+1)(\beta+2)}-\frac{g \mu^{h+2}}{(h+1)(h+2)}\right)+W\right] \\
& \mu \leq t \leq t_{1} \\
& I_{3}(t)=e^{-\alpha t^{\beta}}(a+b \mu)\left(-t-\frac{\alpha t^{\beta+1}}{\beta+1}+t_{2}+\frac{\alpha t_{2}^{\beta+1}}{\beta+1}\right), \quad t_{1} \leq t \leq t_{2} \\
& I_{4}(t)=\frac{(a+b \mu)}{\delta} \log \left(\frac{1+\delta\left(T-t_{2}\right)}{1+\delta(T-t)}\right), \quad t_{2} \leq t \leq T \\
& \Rightarrow e^{-\alpha t_{1}{ }^{\beta}}(a+b \mu)\left(-t_{1}-\frac{\alpha t_{1}{ }^{\beta+1}}{\beta+1}+t_{2}+\frac{\alpha t_{2}{ }^{\beta+1}}{\beta+1}\right)-e^{-\alpha t_{1}{ }^{\beta}+g t_{1}{ }^{h}}\left\{-(a+b \mu)\left(t_{1}+\frac{\alpha t_{1}^{\beta+1}}{\beta+1}-\frac{g t_{1}{ }^{h+1}}{h+1}\right)\right. \\
& \left.+b\left(\frac{\mu^{2}}{2}+\frac{\alpha \mu^{\beta+2}}{(\beta+1)(\beta+2)}-\frac{g \mu^{h+2}}{(h+1)(h+2)}\right)+W\right\}=0
\end{aligned}
$$

Now, the costs contributing the total cost of the system in case 1 are given as follows:

1) Present worth ordering and purchase cost :

$$
P C=c_{o}+c_{p} W
$$

2) Present worth holding cost :

$$
H C=c_{h}\left[\int_{0}^{\mu} I_{1}(t) e^{-r t} d t+\int_{\mu}^{t_{1}} I_{2}(t) e^{-r(\mu+t)} d t+\int_{t_{1}}^{t_{2}} I_{3}(t) e^{-r\left(t_{1}+t\right)} d t\right]
$$

3) Present worth amelioration cost : 


$$
A C=c_{a}\left[\int_{0}^{\mu} g h t^{h-1} I_{1}(t) e^{-r t} d t+\int_{\mu}^{t_{1}} g h t^{h-1} I_{2}(t) e^{-r(\mu+t)} d t\right]
$$

4) Present worth deterioration cost :

$$
D C=c_{d}\left[\int_{0}^{\mu} \alpha \beta t^{\beta-1} I_{1}(t) e^{-r t} d t+\int_{\mu}^{t_{1}} \alpha \beta t^{\beta-1} I_{2}(t) e^{-r(\mu+t)} d t+\int_{t_{1}}^{t_{2}} \alpha \beta t^{\beta-1} I_{3}(t) e^{-r\left(t_{1}+t\right)} d t\right]
$$

5) Present worth shortage cost :

$$
S C=c_{s} \int_{t_{2}}^{T}\left(-I_{4}(t)\right) e^{-r\left(t_{2}+t\right)} d t
$$

6) Present worth lost sales cost :

$$
L C=c_{l} \int_{t_{2}}^{T}\left(1-\frac{1}{1+\delta(T-t)}\right)(a+b \mu) e^{-r\left(t_{2}+t\right)} d t
$$

Our problem is to minimize

$T C_{1}\left(\mu, t_{1}, t_{2}, W\right)=P C+H C+A C+D C+S C+L C$

subject to $\mu<t_{1}<t_{2}<T$

and constraint given by equ.(9).

$\mu, t_{1}, t_{2}, W \geq 0$

Case 2: when $t 1<\mu$

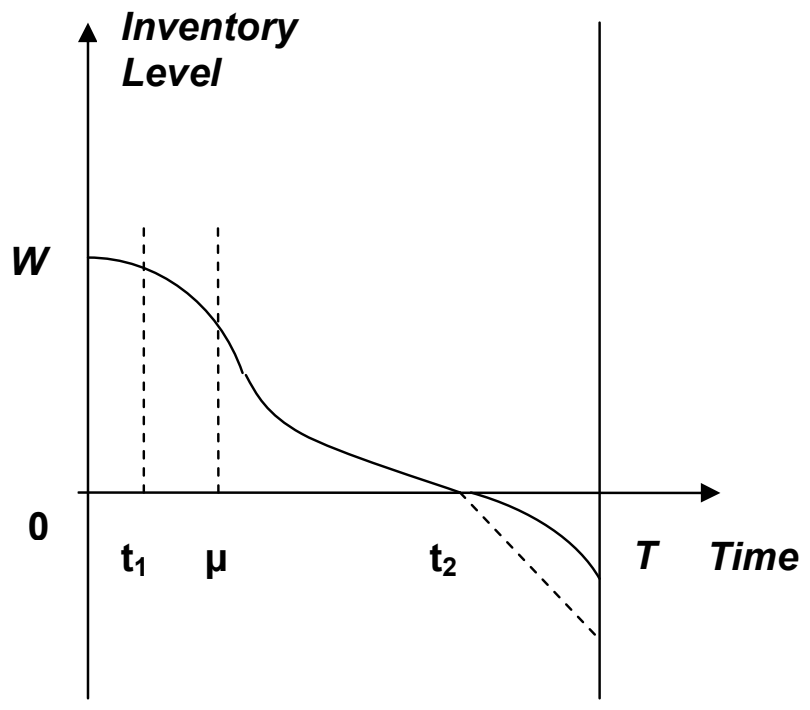

Fig. 2 : Variation of inventory level with time for Case 2

Inventory system can be represented by the following differential equations:

$\frac{d I_{1}(t)}{d t}+\left(\alpha \beta t^{\beta-1}-g h t^{h-1}\right) I_{1}(t)=-(a+b t)$

$0 \leq t \leq t_{1}$

with $I_{1}(0)=W$

$\frac{d I_{2}(t)}{d t}+\alpha \beta t^{\beta-1} I_{2}(t)=-(a+b t)$

$t_{1} \leq t \leq \mu$

with $I_{2}\left(t_{1}\right)=I_{1}\left(t_{1}\right)$

$\frac{d I_{3}(t)}{d t}+\alpha \beta t^{\beta-1} I_{3}(t)=-(a+b \mu)$

$\mu \leq t \leq t_{2}$

with $I_{3}\left(t_{2}\right)=0$ 


$$
\begin{aligned}
& \frac{d I_{4}(t)}{d t}=-\frac{(a+b \mu)}{1+\delta(T-t)} \\
& \text { with } I_{4}\left(t_{2}\right)=0
\end{aligned} \quad t_{2} \leq t \leq T
$$

The solution of the above equations can be given as:

$$
\begin{aligned}
& I_{1}(t)=e^{-\alpha t^{\beta}+g t^{h}}\left[-a\left(t+\frac{\alpha t^{\beta+1}}{\beta+1}-\frac{g t^{h+1}}{h+1}\right)-b\left(\frac{t^{2}}{2}+\frac{\alpha t^{\beta+2}}{\beta+2}-\frac{g t^{h+2}}{h+2}\right)+W\right] \quad 0 \leq t \leq t_{1} \\
& I_{2}(t)=e^{-\alpha t^{\beta}}\left[-(a+b t) \frac{\alpha t^{\beta+1}}{\beta+1}+\frac{b \alpha t^{\beta+2}}{(\beta+1)(\beta+2)}-a t-\frac{b t^{2}}{2}-e^{-g t_{1}{ }^{h}}\left\{a\left(t_{1}+\frac{\alpha t_{1}^{\beta+1}}{\beta+1}-\frac{g t_{1}^{h+1}}{h+1}\right)\right.\right. \\
& \left.\left.+b\left(\frac{t_{1}^{2}}{2}+\frac{\alpha t_{1}^{\beta+2}}{(\beta+2)}-\frac{g t_{1}^{h+2}}{(h+2)}\right)-W\right\}+\left(a+b t_{1}\right) \frac{\alpha t_{1}^{\beta+1}}{\beta+1}-\frac{b \alpha t_{1}^{\beta+2}}{(\beta+1)(\beta+2)}+a t_{1}+\frac{b t_{1}^{2}}{2}\right] \\
& I_{3}(t)=e^{-\alpha t^{\beta}}(a+b \mu)\left(-t-\frac{\alpha t^{\beta+1}}{\beta+1}+t_{2}+\frac{\alpha t_{2}^{\beta+1}}{\beta+1}\right), \quad t_{1} \leq t \leq \mu \\
& I_{4}(t)=\frac{(a+b \mu)}{\delta} \log \left(\frac{1+\delta\left(T-t_{2}\right)}{1+\delta(T-t)}\right), \quad t_{2} \leq t \leq T \\
& \text { Since } I_{3}(\mu)=I_{2}(\mu) \\
& \Rightarrow-(a+b \mu)\left(t_{2}+\frac{\alpha t_{2}^{\beta+1}}{\beta+1}\right)+\frac{b \mu^{2}}{2}-e^{g t_{1}{ }^{h}}\left\{-a\left(t_{1}+\frac{\alpha t_{1}^{\beta+1}}{\beta+1}-\frac{g t_{1}^{h+1}}{h+1}\right)+b\left(\frac{t_{1}^{2}}{2}+\frac{\alpha t_{1}^{\beta+2}}{\beta+2}-\frac{g t_{1}^{h+2}}{h+2}\right)-W\right\} \\
& +\left(a+b t_{1}\right) \frac{\alpha t_{1}^{\beta+1}}{\beta+1}-\frac{b \alpha t_{1}^{\beta+2}}{(\beta+1)(\beta+2)}+a t_{1}+\frac{b t_{1}^{2}}{2}=0
\end{aligned}
$$

Now, the costs contributing the total cost of the system in case 1 are given as follows:

1) Present worth ordering and purchase cost :

$$
P C=c_{o}+c_{p} W
$$

2) Present worth holding cost :

$$
H C=c_{h}\left[\int_{0}^{t_{1}} I_{1}(t) e^{-r t} d t+\int_{t_{1}}^{\mu} I_{2}(t) e^{-r\left(t_{1}+t\right)} d t+\int_{\mu}^{t_{2}} I_{3}(t) e^{-r(\mu+t)} d t\right]
$$

3) Present worth amelioration cost :

$$
A C=c_{a} \int_{0}^{t_{1}} g h t^{h-1} I_{1}(t) e^{-r t} d t
$$

4) Present worth deterioration cost :

$$
D C=c_{d}\left[\int_{0}^{t_{1}} \alpha \beta t^{\beta-1} I_{1}(t) e^{-r t} d t+\int_{t_{1}}^{\mu} \alpha \beta t^{\beta-1} I_{2}(t) e^{-r\left(t_{1}+t\right)} d t+\int_{\mu}^{t_{2}} \alpha \beta t^{\beta-1} I_{3}(t) e^{-r(\mu+t)} d t\right]
$$

5) Present worth shortage cost :

$$
S C=c_{s} \int_{t_{2}}^{T}\left(-I_{4}(t)\right) e^{-r\left(t_{2}+t\right)} d t
$$

6) Present worth lost sales cost :

$$
L C=c_{l} \int_{t_{2}}^{T}\left(1-\frac{1}{1+\delta(T-t)}\right)(a+b \mu) e^{-r\left(t_{2}+t\right)} d t
$$

Our problem is to minimize 
$T C_{2}\left(\mu, t_{1}, t_{2}, W\right)=P C+H C+A C+D C+S C+L C$

subject to $t_{1}<\mu<t_{2}<T$

and constraint given by equ.(25).

$\mu, t_{1}, t_{2}, W \geq 0$

Case 3: when $\mathrm{t} 1<\mathrm{t} 2<\mu$

\section{Inventory \\ Level}

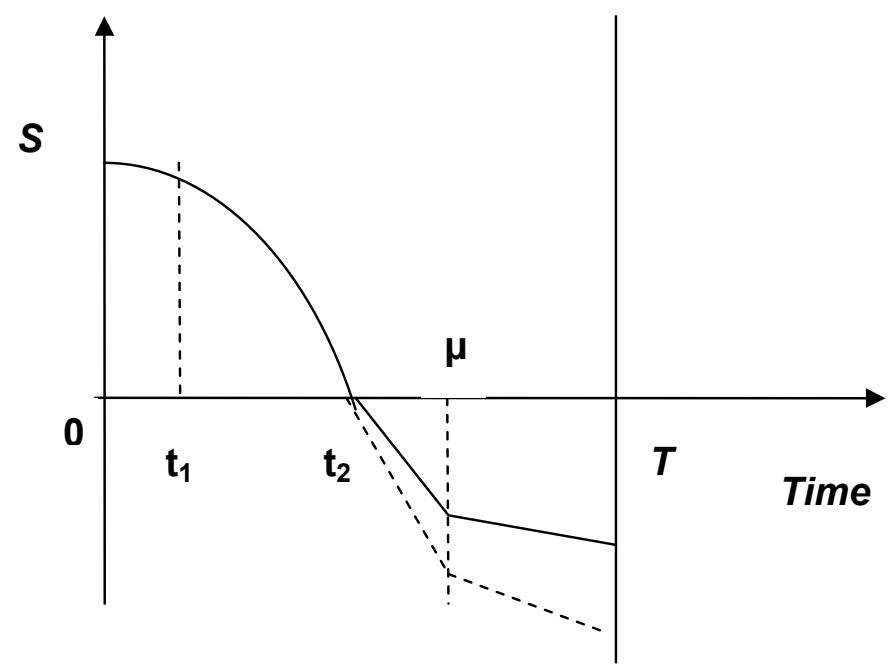

Fig. 3 : Variation of inventory level with time for Case3

Inventory system can be represented by the following differential equations:

$\frac{d I_{1}(t)}{d t}+\left(\alpha \beta t^{\beta-1}-g h t^{h-1}\right) I_{1}(t)=-(a+b t)$

$0 \leq t \leq t_{1}$

with $I_{1}(0)=W$

$\frac{d I_{2}(t)}{d t}+\alpha \beta t^{\beta-1} I_{2}(t)=-(a+b t)$

$$
t_{1} \leq t \leq t_{2}
$$

with $I_{2}\left(t_{2}\right)=0$

$\frac{d I_{3}(t)}{d t}=-\frac{(a+b t)}{1+\delta(T-t)}$

$$
t_{2} \leq t \leq \mu
$$

with $I_{3}\left(t_{2}\right)=0$

$\frac{d I_{4}(t)}{d t}=-\frac{(a+b \mu)}{1+\delta(T-t)}$

$$
\mu \leq t \leq T
$$

with $I_{4}(\mu)=I_{3}(\mu)$

The solution of the above equations can be given as:

$I_{1}(t)=e^{-\alpha t^{\beta}+g t^{h}}\left[-a\left(t+\frac{\alpha t^{\beta+1}}{\beta+1}-\frac{g t^{h+1}}{h+1}\right)-b\left(\frac{t^{2}}{2}+\frac{\alpha t^{\beta+2}}{\beta+2}-\frac{g t^{h+2}}{h+2}\right)+W\right] \quad 0 \leq t \leq t_{1}$ 


$$
\begin{aligned}
& I_{2}(t)=e^{-\alpha t^{\beta}}\left[-(a+b t) \frac{\alpha t^{\beta+1}}{\beta+1}+\frac{b \alpha t^{\beta+2}}{(\beta+1)(\beta+2)}-a t-\frac{b t^{2}}{2}+\left(a+b t_{2}\right) \frac{\alpha t_{2}{ }^{\beta+1}}{\beta+1}-\frac{b \alpha t_{2}{ }^{\beta+2}}{(\beta+1)(\beta+2)}+a t_{2}+\frac{b t_{2}{ }^{2}}{2}\right] \\
& I_{3}(t)=\frac{1}{\delta}\left\{a+b\left(T+\frac{1}{\delta}\right)\right\} \log \left(\frac{1+\delta(T-t)}{1+\delta\left(T-t_{2}\right)}\right)+\frac{b}{\delta^{2}}\left(t-t_{2}\right) \\
& I_{4}(t)=\frac{(a+b \mu)}{\delta} \log \left(\frac{1+\delta(T-t)}{1+\delta(T-\mu)}\right)+\frac{1}{\delta}\left\{a+b\left(T+\frac{1}{\delta}\right)\right\} \log \left(\frac{1+\delta(T-\mu)}{1+\delta\left(T-t_{2}\right)}\right)+\frac{b}{\delta^{2}}\left(\mu-t_{2}\right) \\
& t_{2} \leq t \leq T
\end{aligned}
$$

Since $I_{1}\left(t_{1}\right)=I_{2}\left(t_{1}\right)$

$\Rightarrow e^{g t_{1}^{h}}\left\{-a\left(t_{1}+\frac{\alpha t_{1}^{\beta+1}}{\beta+1}-\frac{g t_{1}^{h+1}}{h+1}\right)-b\left(\frac{t_{1}^{2}}{2}+\frac{\alpha t_{1}^{\beta+2}}{\beta+2}-\frac{g t_{1}^{h+2}}{h+2}\right)+W\right\}+\left(a+b t_{1}\right) \frac{\alpha t_{1}^{\beta+1}}{\beta+1}-\frac{b \alpha t_{1}^{\beta+2}}{(\beta+1)(\beta+2)}$

$+a t_{1}+\frac{b t_{1}^{2}}{2}-\left(a+b t_{2}\right) \frac{\alpha t_{2}^{\beta+1}}{\beta+1}+\frac{b \alpha t_{2}^{\beta+2}}{(\beta+1)(\beta+2)}-a t_{2}-\frac{b t_{2}^{2}}{2}=0$

Now, the costs contributing the total cost of the system in case 1 are given as follows:

1) Present worth ordering and purchase cost :

$$
P C=c_{o}+c_{p} W
$$

2) Present worth holding cost :

$$
H C=c_{h}\left[\int_{0}^{t_{1}} I_{1}(t) e^{-r t} d t+\int_{t_{1}}^{t_{2}} I_{2}(t) e^{-r\left(t_{1}+t\right)} d t\right]
$$

3) Present worth amelioration cost :

$$
A C=c_{a} \int_{0}^{t_{1}} g h t^{h-1} I_{1}(t) e^{-r t} d t
$$

4) Present worth deterioration cost :

$$
D C=c_{d}\left[\int_{0}^{t_{1}} \alpha \beta t^{\beta-1} I_{1}(t) e^{-r t} d t+\int_{t_{1}}^{t_{2}} \alpha \beta t^{\beta-1} I_{2}(t) e^{-r\left(t_{1}+t\right)} d t\right]
$$

5) Present worth shortage cost :

$$
S C=c_{S}\left[\int_{t_{2}}^{\mu}\left(-I_{3}(t)\right) e^{-r(\mu+t)} d t+\int_{t_{2}}^{T}\left(-I_{4}(t)\right) e^{-r\left(t_{2}+t\right)} d t\right]
$$

6) Present worth lost sales cost :

$$
L C=c_{l}\left[\int_{t_{2}}^{\mu}\left(1-\frac{1}{1+\delta(T-t)}\right)(a+b t) e^{-r\left(t_{2}+t\right)} d t+\int_{\mu}^{T}\left(1-\frac{1}{1+\delta(T-t)}\right)(a+b \mu) e^{-r(\mu+t)} d t\right]
$$

Our problem is to minimize

$T C_{2}\left(\mu, t_{1}, t_{2}, W\right)=P C+H C+A C+D C+S C+L C$

subject to $t_{1}<t_{2}<\mu<T$

and constraint given by equ.(41).

$\mu, t_{1}, t_{2}, W \geq 0$

\section{GENETIC ALGORITHM}

Genetic Algorithm was developed by Holland and his colleagues in the 1960s and 1970s. Genetic Algorithms are inspired by the evolutionist theory explaining the origin of species. In nature, weak and unfit species within their environment are faced with extinction by natural selection. The strong ones have greater opportunity to pass their genes to future generations via reproduction. In the long run, species carrying the correct combination in their genes become dominant in their population. Sometimes, during the slow 
process of evolution, random changes may occur in genes. If these changes provide additional advantages in the challenge for survival, new species evolve from the old ones. Unsuccessful changes are eliminated by natural selection.

GA terminology, a solution vector $\mathrm{x} € \mathrm{X}$ is called an individual or a chromosome. Chromosomes are made of discrete units called genes. Each gene controls one or more features of the chromosome. In the original implementation of GA by Holland, genes are assumed to be binary digits. In later implementations, more varied gene types have been introduced. Normally, a chromosome corresponds to a unique solution $\mathrm{x}$ in the solution space. This requires a mapping mechanism between the solution space and the chromosomes. This mapping is called an encoding. In fact, GA works on the encoding of a problem, not on the problem itself.

GA operates with a collection of chromosomes, called a population. The population is normally randomly initialized. As the search evolves, the population includes fitter and fitter solutions, and eventually it converges, meaning that it is dominated by a single solution. Holland also presented a proof of convergence (the schema theorem) to the global optimum where chromosomes are binary vectors.

GA use two operators to generate new solutions from existing ones: crossover and mutation. The crossover operator is the most important operator of GA. In crossover, generally two chromosomes, called parents, are combined together to form new chromosomes, called offspring. The parents are selected among existing chromosomes in the population with preference towards fitness so that offspring is expected to inherit good genes which make the parents fitter. By iteratively applying the crossover operator, genes of good chromosomes are expected to appear more frequently in the population, eventually leading to convergence to an overall good solution.
The mutation operator introduces random changes into characteristics of chromosomes. Mutation is generally applied at the gene level. In typical GA implementations, the mutation rate (probability of changing the properties of a gene) is very small and depends on the length of the chromosome. Therefore, the new chromosome produced by mutation will not be very different from the original one. Mutation plays a critical role in GA. As discussed earlier, crossover leads the population to converge by making the chromosomes in the population alike. Mutation reintroduces genetic diversity back into the population and assists the search escape from local optima. Reproduction involves selection of chromosomes for the next generation. In the most general case, the fitness of an individual determines the probability of its survival for the next generation.

A more complete discussion of GAs including extensions to the general algorithm and related topics can be found in books by Davis (1991),, Holland (1975), Michalewicz (1994) and Goldberg (1989).

\section{NUMERICAL EXAMPLE}

In this section, we solved a numerical example of the proposed model using the above-described GA. for running GA, we set the population size equal to 20 , elite count equal to 2, crossover fraction equal to 0.8 with Gaussian mutation function and the stopping criteria include the maximal iterations of each cycle is 50 .

\subsection{Case1 when $t_{1}>\mu$}

We consider the values of the parameters in appropriate units such that $\mathrm{a}=2, \mathrm{~b}=1, \alpha=0.03, \beta=2.2, \mathrm{~g}=0.4, \mathrm{~h}=0.5, \delta=0.06$, $\mathrm{r}=0.06, \mathrm{c}_{\mathrm{o}}=5, \mathrm{c}_{\mathrm{p}}=1, \mathrm{c}_{\mathrm{h}}=0.5, \mathrm{c}_{\mathrm{a}}=1.5, \mathrm{c}_{\mathrm{d}}=5, \mathrm{c}_{\mathrm{s}}=1, \mathrm{c}_{1}=2, \mathrm{~T}$ $=1$.Optimal value of $\mathrm{t}_{1}=0.84711, \mu=0.07518, \mathrm{t}_{2}=0.9396$, $\mathrm{W}=1.47204$. The minimized total cost $\mathrm{TC}_{1}=7.46853055$ is calculated from equ. (16).

\subsubsection{Sensitivity Analysis}

Table 1.Sensitivity analsysis based on example when case 1 with different parameters.

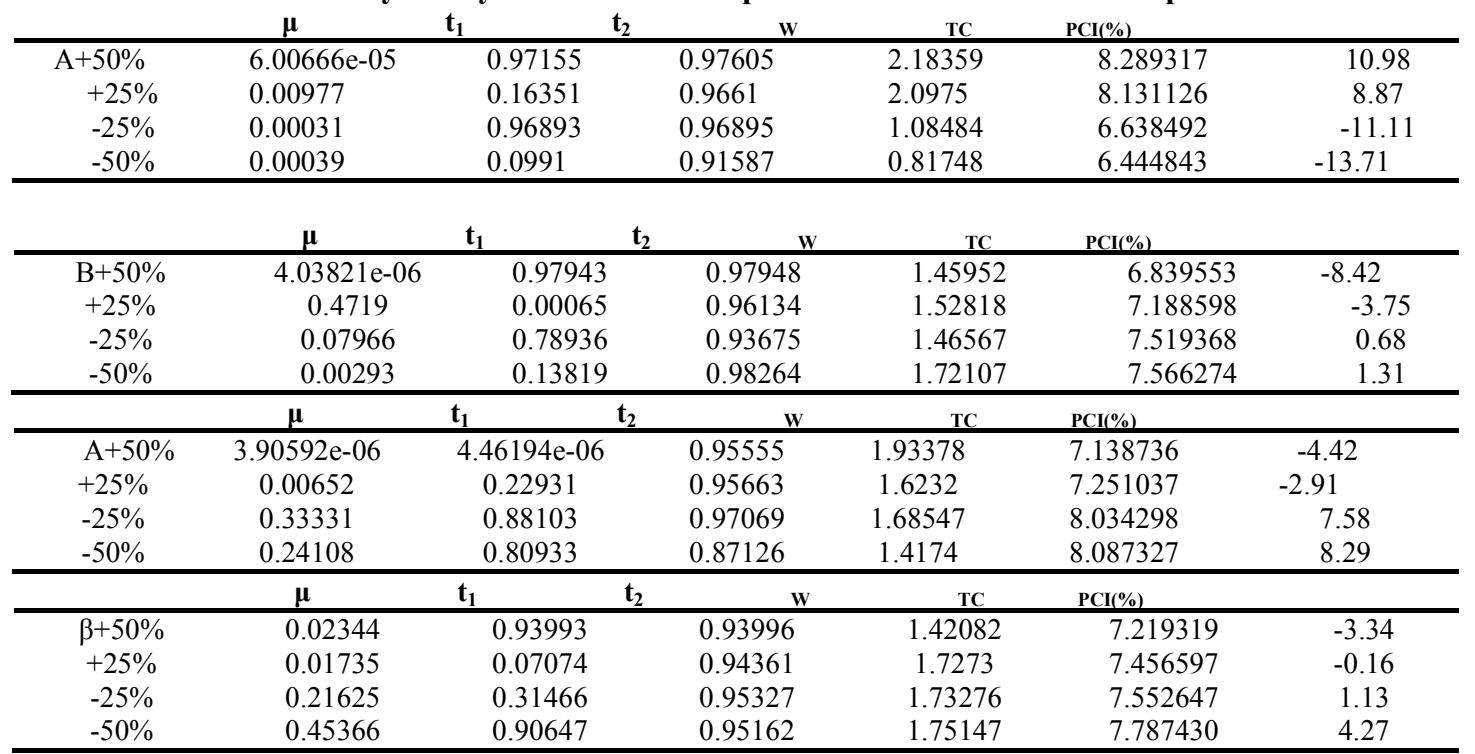




\begin{tabular}{|c|c|c|c|c|c|c|}
\hline & $\mu$ & $\mathbf{t}_{1}$ & w & $\mathrm{TC}$ & PCI $(\%)$ & \\
\hline $\mathrm{g}+50 \%$ & $3.02792 \mathrm{e}-05$ & 0.99344 & 0.99354 & 1.21323 & 6.500563 & -12.96 \\
\hline$+25 \%$ & 0.01414 & 0.84566 & 0.92738 & 1.29845 & 7.154485 & -4.20 \\
\hline$-25 \%$ & 0.0002 & 0.16977 & 0.91043 & 1.63309 & 7.549776 & 1.09 \\
\hline \multirow[t]{2}{*}{$-50 \%$} & 0.2439 & 0.49396 & 0.93073 & 1.85084 & 7.905908 & 5.86 \\
\hline & $\mu$ & $t_{1}$ & $\mathbf{w}$ & $\mathrm{TC}$ & PCI(\%) & \\
\hline $\mathrm{h}+50 \%$ & 0.00011 & 0.97965 & 0.97967 & 1.53598 & 6.905063 & -7.54 \\
\hline$+25 \%$ & 0.01316 & 0.35634 & 0.95371 & 1.61284 & 7.248990 & -2.94 \\
\hline$-25 \%$ & 0.28562 & 0.71165 & 0.92374 & 1.55901 & 7.544445 & 1.02 \\
\hline \multirow[t]{2}{*}{$-50 \%$} & 0.37454 & 0.87149 & 0.94055 & 1.56625 & 7.577209 & 1.46 \\
\hline & $\mu$ & $t_{1}$ & w & $\mathrm{TC}$ & PCI $(\%)$ & \\
\hline$\delta+50 \%$ & 0.44958 & 0.90221 & 0.974 & 1.7781 & 7.667156 & 2.66 \\
\hline$+25 \%$ & 0.01205 & 0.51896 & 0.94424 & 1.49733 & 7.611114 & 1.91 \\
\hline$-25 \%$ & $2.28638 \mathrm{e}-08$ & 0.97083 & 0.97083 & 1.44855 & 7.185103 & -3.80 \\
\hline \multirow[t]{2}{*}{$-50 \%$} & $1.39129 \mathrm{e}-08$ & $1.39129 \mathrm{e}-08$ & 0.95972 & 1.93578 & 7.090317 & -5.06 \\
\hline & $\mu$ & $t_{1}$ & w & $\mathrm{TC}$ & PCI $(\%)$ & \\
\hline $\mathrm{r}+50 \%$ & 0.50334 & 0.79236 & 0.92743 & 1.75674 & 7.858291 & 5.22 \\
\hline$+25 \%$ & 0.02183 & 0.79174 & 0.82668 & 1.28015 & 7.703888 & 3.15 \\
\hline$-25 \%$ & 0.00739 & 0.33988 & 0.92434 & 1.51709 & 7.317177 & -2.03 \\
\hline \multirow[t]{2}{*}{$-50 \%$} & $4.5438 \mathrm{e}-06$ & 0.98539 & 0.98541 & 1.46701 & 7.143026 & -4.36 \\
\hline & $\mu$ & $t_{1}$ & w & $\mathrm{TC}$ & PCI $\%)$ & \\
\hline $\mathrm{c}_{\mathrm{o}}+50 \%$ & 0 & 0.96782 & 0.96794 & 1.4449 & 9.685661 & 29.69 \\
\hline$+25 \%$ & 0.0063 & 0.85289 & 0.95859 & 1.45114 & 8.585960 & 14.96 \\
\hline$-25 \%$ & 0.00098 & 0.24474 & 0.96567 & 1.62248 & 6.364058 & -14.79 \\
\hline \multirow[t]{2}{*}{$-50 \%$} & $1.55578 \mathrm{e}-07$ & 7.18733e-05 & 0.95649 & 1.92271 & 4.603603 & -38.36 \\
\hline & $\mu$ & $t_{1}$ & w & $\mathrm{TC}$ & PCI $(\%)$ & \\
\hline$c_{p}+50 \%$ & 0.21167 & 0.90572 & 0.96967 & 1.60035 & 8.487937 & 13.65 \\
\hline$+25 \%$ & 0.0215 & 0.97365 & 0.97491 & 1.46932 & 7.592718 & 1.66 \\
\hline$-25 \%$ & 0.49731 & 0.91255 & 0.99094 & 1.84358 & 7.340344 & -1.72 \\
\hline \multirow[t]{2}{*}{$-50 \%$} & 0.02247 & 0.05619 & 0.9401 & 1.74636 & 6.581384 & -11.88 \\
\hline & $\mu$ & $t_{1}$ & w & $\mathrm{TC}$ & PCI $\%$ ) & \\
\hline $\mathrm{c}_{\mathrm{h}}+50 \%$ & 0.55898 & 0.57701 & 0.96509 & 1.9233 & 8.314752 & 11.33 \\
\hline$+25 \%$ & 0.21772 & 0.25494 & 0.94707 & 1.74881 & 7.565647 & 1.300 \\
\hline$-25 \%$ & 0.00147 & 0.95036 & 0.95231 & 1.42629 & 7.147677 & -4.30 \\
\hline \multirow[t]{2}{*}{$-50 \%$} & 0.00864 & 0.77702 & 0.97352 & 1.4859 & 6.938663 & -7.09 \\
\hline & $\mu$ & $t_{1}$ & $\mathbf{w}$ & $\mathrm{TC}$ & PCI $\%$ & \\
\hline $\mathrm{c}_{\mathrm{s}}+50 \%$ & 0.14844 & 0.59337 & 0.87545 & 1.46359 & 7.568550 & 1.34 \\
\hline$+25 \%$ & 0.42853 & 0.95924 & 0.97628 & 1.757 & 7.559448 & 1.22 \\
\hline$-25 \%$ & $6.27729 \mathrm{e}-05$ & 0.97036 & 0.97754 & 1.45795 & 7.196516 & -3.64 \\
\hline \multirow[t]{2}{*}{$-50 \%$} & $1.32919 \mathrm{e}-08$ & $1.32919 \mathrm{e}-08$ & 0.95975 & 1.93586 & 7.090379 & -5.06 \\
\hline & $\mu$ & $t_{1}$ & w & $\mathrm{TC}$ & PCI $\%$ & \\
\hline $\mathrm{c}_{\mathrm{d}}+50 \%$ & 0.32072 & 0.60551 & 0.97339 & 1.74188 & 7.645105 & 2.36 \\
\hline$+25 \%$ & 0.0433 & 0.77153 & 0.83073 & 1.30159 & 7.579308 & 1.48 \\
\hline$-25 \%$ & 0 & $3.35834 \mathrm{e}-09$ & 0.96081 & 1.93808 & 7.070836 & -5.32 \\
\hline \multirow[t]{2}{*}{$-50 \%$} & 0 & 0 & 0.96189 & 1.94034 & 7.051183 & -5.59 \\
\hline & $\mu$ & $t_{1}$ & w & $\mathrm{TC}$ & PCI $(\%)$ & \\
\hline$c_{1}+50 \%$ & 0.71312 & 0.80305 & 0.97073 & 2.01847 & 8.511481 & 13.96 \\
\hline$+25 \%$ & 0.3503 & 0.84224 & 0.97325 & 1.71287 & 7.524059 & 0.74 \\
\hline$-25 \%$ & 0.00027 & 0.96311 & 0.96313 & 1.43897 & 7.177566 & -3.90 \\
\hline \multirow[t]{2}{*}{$-50 \%$} & $9.94633 \mathrm{e}-08$ & 0.95032 & 0.95033 & 1.42249 & 7.161515 & -4.11 \\
\hline & $\mu$ & $t_{1}$ & w & TC & PCI $\%$ & \\
\hline $\mathrm{c}_{\mathrm{a}}+50 \%$ & 0.09693 & 0.38251 & 0.94755 & 1.60535 & 8.278469 & 10.84 \\
\hline$+25 \%$ & 0.03209 & 0.17487 & 0.9279 & 1.62255 & 7.828476 & 4.82 \\
\hline$-25 \%$ & 0.00914 & 0.02502 & 0.929 & 1.76695 & 7.270120 & -2.66 \\
\hline$-50 \%$ & 0.00091 & 0.97647 & 0.97838 & 1.454 & 6.842726 & -8.38 \\
\hline
\end{tabular}

5.2 Case 2 when $\mathrm{t}_{1}<\mu$

We consider the values of the parameters in appropriate units such that $\mathrm{a}=10, \mathrm{~b}=2, \alpha=0.02, \beta=3.2, \mathrm{~g}=0.4, \mathrm{~h}=0.5, \delta=0.16, \mathrm{r}=0.06, \mathrm{c}_{\mathrm{o}}=5$, $\mathrm{c}_{\mathrm{p}}=1, \mathrm{c}_{\mathrm{h}}=3, \mathrm{c}_{\mathrm{s}}=1, \mathrm{c}_{\mathrm{d}}=5, \mathrm{c}_{1}=2, \mathrm{c}_{\mathrm{a}}=3, \mathrm{~T}=1$. Optimal value of $\mathrm{t}_{1}$, i.e., $\mathrm{t}_{1} *=0.002, \mu=0.05766, \mathrm{t}_{2}=0.08977, \mathrm{~W}=0.89509$. The minimized total cost $\mathrm{TC}_{2}=3.110885$ is calculated from equ.(32). 
5.2.1 Sensitivity Analysis

Table 2 .Sensitivity analsysis based on example when case 2 with different parameters.

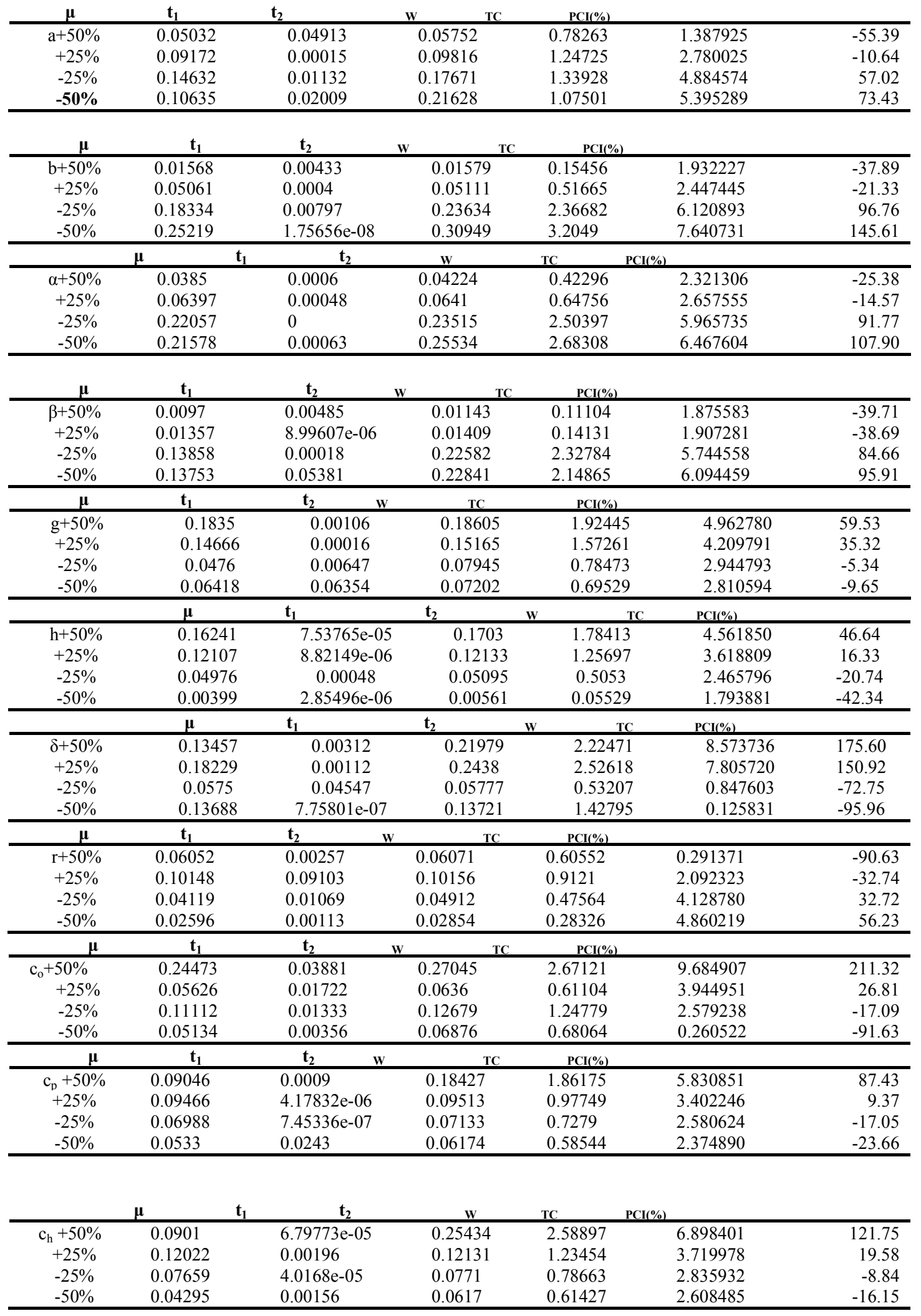




\begin{tabular}{|c|c|c|c|c|c|c|}
\hline $\boldsymbol{\mu}$ & $t_{1}$ & $\mathbf{t}_{2}$ & $\mathrm{TC}$ & PCI(\%) & & \\
\hline $\mathrm{c}_{\mathrm{s}}+50 \%$ & 0.09236 & 0.05854 & 0.10798 & 0.99612 & 1.803250 & -42.03 \\
\hline$+25 \%$ & 0.08271 & 0.01522 & 0.08335 & 0.81115 & 2.146414 & -31.00 \\
\hline$-25 \%$ & 0.03461 & 0.01074 & 0.03465 & 0.33469 & 3.159460 & 1.56 \\
\hline$-50 \%$ & 0.0772 & 0.01336 & 0.08218 & 0.80089 & 4.773249 & 53.44 \\
\hline$\mu$ & $t_{1}$ & $t_{2}$ & w & PCII\% & & \\
\hline $\mathrm{c}_{\mathrm{d}}+50 \%$ & 0.20871 & 0.15781 & 0.21498 & 1.92602 & 5.805854 & -55.39 \\
\hline$+25 \%$ & 0.14424 & 0.02775 & 0.17884 & 1.73677 & 4.946861 & 59.02 \\
\hline$-25 \%$ & 0.0777 & 0.06839 & 0.08634 & 1.78344 & 3.087571 & -0.75 \\
\hline$-50 \%$ & 0.05905 & 0.00351 & 0.05909 & 1.58706 & 2.594540 & -16.60 \\
\hline$\mu$ & $t_{1}$ & $t_{2}$ & w & PCI $\%$ & & \\
\hline $\mathrm{c}_{1}+50 \%$ & 0.20539 & 0.00309 & 0.24114 & 2.49648 & 6.383779 & 105.21 \\
\hline$+25 \%$ & 0.11426 & 0.10576 & 0.11605 & 1.03625 & 3.747681 & 20.47 \\
\hline$-25 \%$ & 0.05053 & 0.01751 & 0.05142 & 0.49275 & 2.349360 & -24.48 \\
\hline$-50 \%$ & 0.05561 & 0.00015 & 0.05699 & 0.57655 & 2.262828 & -27.26 \\
\hline$\mu$ & $\mathbf{t}_{1}$ & $t_{2}$ & $\mathrm{TC}$ & PCI & & \\
\hline $\mathrm{c}_{\mathrm{a}}+50 \%$ & 0.13359 & 0.00137 & 0.16688 & 1.70578 & 4.580843 & 47.25 \\
\hline$+25 \%$ & 0.10618 & 0.00094 & 0.15469 & 1.57162 & 4.308193 & 38.49 \\
\hline$-25 \%$ & 0.08101 & 0.02322 & 0.08407 & 0.80668 & 3.017143 & -3.01 \\
\hline$-50 \%$ & 0.04898 & 0.00079 & 0.06058 & 0.60727 & 2.600193 & -16.42 \\
\hline
\end{tabular}

\subsection{Case 3 when $\mathrm{t}_{1}<\mathrm{t}_{2}<\mu$}

We consider the values of the parameters in appropriate units such that $\mathrm{a}=10, \mathrm{~b}=0.5, \alpha=0.04, \beta=2.2, \mathrm{~g}=0.4, \mathrm{~h}=0.5, \delta=0.16, \mathrm{r}=0.6, \mathrm{c}_{\mathrm{o}}=5$, $\mathrm{c}_{\mathrm{p}}=1, \mathrm{c}_{\mathrm{h}}=3, \mathrm{c}_{\mathrm{s}}=1, \mathrm{c}_{\mathrm{d}}=5, \mathrm{c}_{1}=2, \mathrm{c}_{\mathrm{a}}=3, \mathrm{~T}=1$. Optimal value of $\mathrm{t}_{1}$, i.e., $\mathrm{t}_{1}{ }^{*}=0.06315, \mu=1, \mathrm{t}_{2}=0.14019, \mathrm{~W}=1.29054$. The minimized total cost $\mathrm{TC}_{3}=1.205013$ is calculated from equ.(48).

\subsubsection{Sensitivity Analysis:}

Table 3.Sensitivity analsysis based on example when case 3with different parameters.

\begin{tabular}{|c|c|c|c|c|c|c|}
\hline$\mu$ & $t_{1}$ & $t_{2}$ & w & $\mathrm{TC}$ & CI (\%) & \\
\hline$a+50 \%$ & 1 & $4.56439 \mathrm{e}-08$ & 0.19287 & 2.90307 & 2.522166 & 109.31 \\
\hline$+25 \%$ & 1 & 0.01167 & 0.17841 & 2.14603 & 2.236127 & 85.57 \\
\hline$-25 \%$ & 1 & 0.00092 & 0.09184 & 0.6825 & 0.126330 & -89.52 \\
\hline$-50 \%$ & 0.9995 & 0.00057 & 0.00801 & 0.0397 & -0.997823 & -182.81 \\
\hline 1 & $t_{1}$ & $\mathbf{t}_{2}$ & w & $\mathrm{TC}$ & PCI(\%) & \\
\hline $\mathrm{b}+50 \%$ & 1 & 0.04091 & 0.07777 & 0.72915 & -2.404196 & -299.52 \\
\hline$+25 \%$ & 1 & 0.00093 & 0.084 & 0.8321 & -1.567898 & -230.11 \\
\hline$-25 \%$ & 0.99983 & 0.01623 & 0.14734 & 1.40685 & 1.915507 & 58.96 \\
\hline$-50 \%$ & 1 & 0.03053 & 0.14734 & 1.38308 & 2.504718 & 107.86 \\
\hline$\mu$ & $\mathbf{t}_{1}$ & $t_{2}$ & w & PCI $\%$ ) & & \\
\hline$\alpha+50 \%$ & 1 & 0.04561 & 0.07492 & 0.70055 & -1.136903 & -194.35 \\
\hline$+25 \%$ & 1 & 0.12413 & 0.13227 & 1.19949 & 0.952947 & -20.99 \\
\hline$-25 \%$ & 1 & 0.00069 & 0.34518 & 3.4482 & 7.564523 & 527.75 \\
\hline$-50 \%$ & 0.99986 & $4.15431 \mathrm{e}-14$ & 0.53191 & 5.39746 & 13.169198 & 992.87 \\
\hline$\mu$ & $t_{1}$ & $\mathbf{t}_{2}$ & $\mathrm{TC}$ & PCI $(\%)$ & & \\
\hline$\beta+50 \%$ & 1 & 0.23886 & 0.27459 & 2.38726 & 5.905424 & 390.07 \\
\hline$+25 \%$ & 1 & 0.00054 & 0.1926 & 1.91759 & 2.740872 & 127.46 \\
\hline$-25 \%$ & 1 & 0.01135 & 0.03668 & 0.35337 & -2.558105 & -312.29 \\
\hline$-50 \%$ & 1 & 0.00093 & 0.00694 & 0.06858 & -3.667661 & -404.37 \\
\hline$\mu$ & $t_{1}$ & $t_{2}$ & $w$ & PCI $(\%)$ & & \\
\hline $\mathrm{g}+50 \%$ & 1 & $1.58187 \mathrm{e}-05$ & 0.00107 & 0.01065 & -3.886639 & -422.54 \\
\hline$+25 \%$ & 1 & $2.43071 \mathrm{e}-05$ & 0.08564 & 0.85614 & -0.856076 & -171.04 \\
\hline$-25 \%$ & 0.99976 & $8.11067 \mathrm{e}-10$ & 0.24193 & 2.43515 & 4.286349 & 255.71 \\
\hline$-50 \%$ & 1 & $4.52692 \mathrm{e}-05$ & 0.34688 & 3.49821 & 7.560138 & 527.39 \\
\hline $\boldsymbol{\mu}$ & $t_{1}$ & $t_{2}$ & $\begin{array}{ll}\text { W } & \text { TC } \\
\end{array}$ & PCI(\%) & & \\
\hline $\mathrm{h}+50 \%$ & 1 & 0.06837 & 0.33334 & 3.20416 & 7.393521 & 513.56 \\
\hline$+25 \%$ & 1 & 0.00019 & 0.18805 & 1.88634 & 2.567359 & 113.06 \\
\hline$-25 \%$ & 1 & 0.12084 & 0.12522 & 1.08929 & 0.772998 & -35.85 \\
\hline$-50 \%$ & 1 & 0.00046 & 0.06628 & 0.62628 & -1.458916 & -221.07 \\
\hline
\end{tabular}




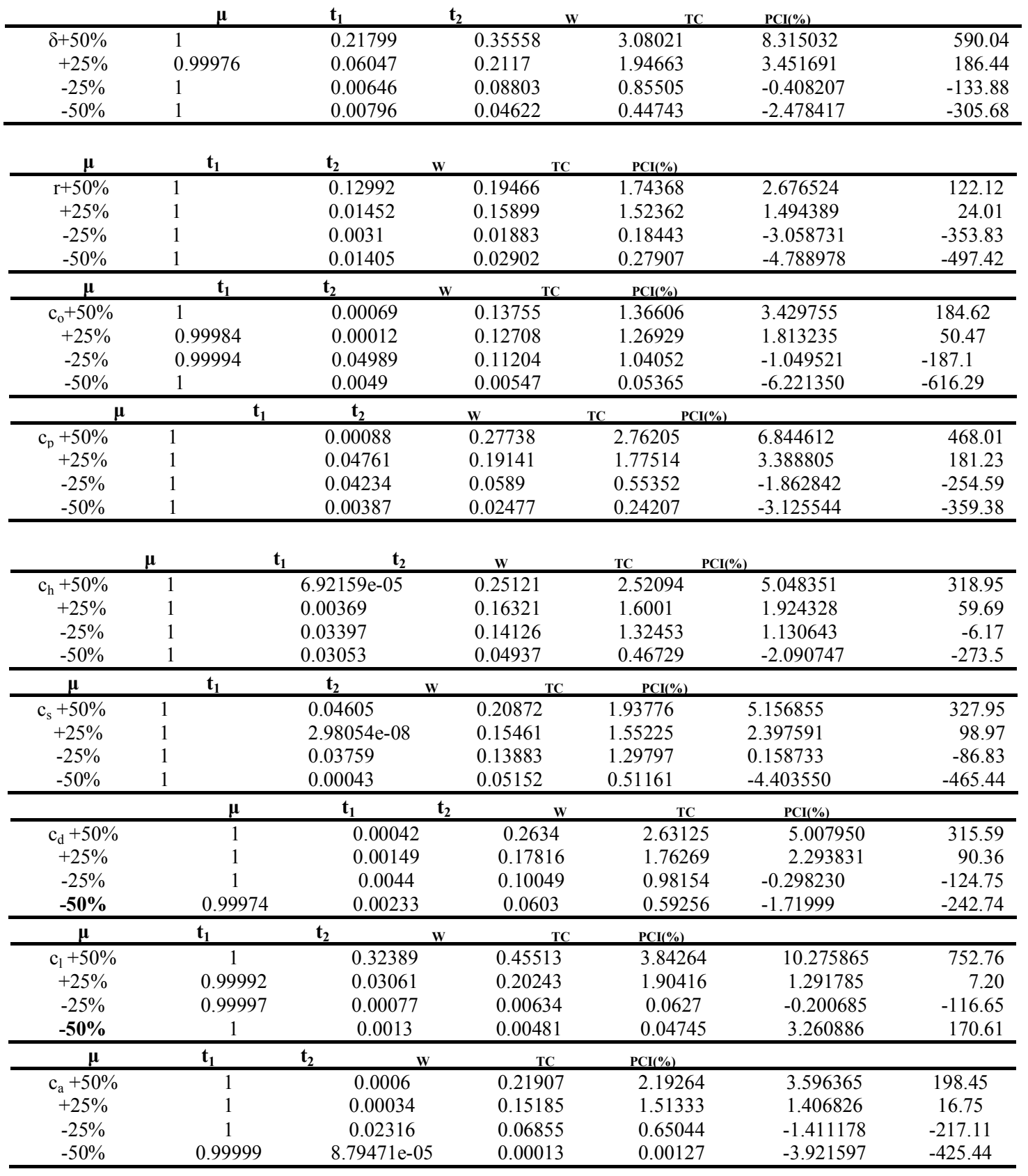

\section{CONCLUSION}

This work is an attempt for analyzing an order level inventory model for ameliorating items. The analysis helps us formulate a generic model for further work. Hence, all the efforts have been carefully directed towards the possible futuristic enhancements of the model. The demand rate has been generically selected to represent any function of time till the stabilization instant arrives (general ramp type demand rate), and the backlogging rate has been generically chosen to represent any non-increasing function of the waiting time, up to the next replenishment. The inventory model has been analyzed for the scenarios of replenishment policy, starting with no shortages. The optimal replenishment policy for the model is derived for the above mentioned inventory system. A numerical example is also presented to illustrate this model.

\section{REFERENCES}

[1] Skouri, K. and Papachristos, S. (2003), "Optimal stopping and restarting production times for an EOQ model with deteriorating items and time-dependent partial backlogging", International Journal of Production Economics, Vol.81-82, pp.525-531.

[2] Panda, S., Senapati, S. and Basu, M. (2008). “Optimal replenishment policy for perishable seasonal products in a season with ramp-type time dependent demand". Computers \& Industrial Engineering 54, 301-314.

[3] Benkherouf, L. and Balkhi, Zaid T. (1997). "On an Inventory Model for Deteriorating Items and Time- 
Varying Demand." Mathematical Methods of Operations Research,45,221-233.

[4] Chakrabarty, T., Giri, B.C. and Chauduri, K.S. (1997). "An EOQ model for items with Weibull distribution deterioration, shortages and trended demand: An Extension of Philip's model." Computers Ops. Res. ,25, 7/8,649-657.

[5] S1la, C., M. Fatih and L. Chung-Yee (2005). "A comparison of out bound dispatch policies for integrated inventory and transportation decisions." European Journal of Operational Research.

[6] Jen-Ming, C. and C. Tsung-Hui (2005). "The multi-item replenishment problem in a two-echelon supply chain: the effect of centralization versus decentralization." Computers \& Operations Research 32.

[7] Bahloul, K., A. Baboli and J.-P. Campagne (2008). "Optimization methods for inventory and transportation problem in Supply Chain: literature review." International Conference on Information Systems, Logistics and Supply Chain (ILS). Madison, WI, U.S.A.

[8] Datta, T.K., and Pal, A.K. (1991). "Effects of inflation and Time-value of money on an inventory model with linear time-dependent demand rate and shortages." European Journal of Operational Research ,52,326-333.

[9] Datta, T.P., and Pal, A.K. (1988). "Order level inventory system with power demand patterns for items with variable rate of deterioration." Indian $J$ of pure App. Math.,19(11);1043-1053.

[10] Ghare, P. M., \& Schrader, G. F. (1963). "A model for an exponentially decaying inventory." Journal of Industrial Engineering, 14, 238-243.

[11] Goyal S.K. (1987). "Economic ordering policies for deteriorating items over an infinite time horizon." European Journal of Operational Research, 28, 298-301.

[12] Hariga, M. (1995). "Effects of inflation and time-value of money on an inventory model with time-dependent demand rate and shortages." European Journal of Operational Research,81,512-520.

[13] Hariga, M. (1997). "Optimal inventory policies for perishable items with time-dependent demand." Int. J. Production Economics,50,35-41.

[14] Hill, R.M. (1995). "Inventory model for increasing demand followed by level demand." Journal of the Operational Research Society 46, 1250-1259.

[15] Silver E. A. and Meal, H. C., 1969. A simple modification of the EOQ for the case of a varying demand rate. Production of Inventory Management.10 (4), 52-65.

[16] Hwang, H. S. (1997). "A study on an inventory model for items with Weibull ameliorating." Computers and Industrial Engineering, 33, 701-704.

[17] Hwang, H. S. (1999). "Inventory models for both deteriorating and ameliorating items." Computers and Industrial Engineering, 37, 257-260.
[18] Lin, Y. and Lin, C. (2006). "Purchasing model for deteriorating items with time-varying demand under inflation and time discounting." ,Int J Adv Manuf. Technology 27: 816-823.

[19] Mondal, B., \& Pal, A. K. (1998). "Order level inventory system with ramp type demand for deteriorating items." Journal of Interdisciplinary Mathematics, 1, 49-66.

[20] Moon, I., Giri, B.C., Ko, B.(2005). "Economic order quantity models for ameliorating/deteriorating items under inflation and time discounting." European Journal of Operational Research 162, 773-785.

[21] Chih-Yao Lo, (2008). "Advance of Dynamic ProductionInventory Strategy for Multiple Policies using Genetic Algorithm”, Information Technology Journal, Vol. 7, pp. 647-653..

[22] Skouri, K. , Konstantaras, I., Papachristos, S., Ganas, I.(2009). "Inventory models with ramp type demand rate, partial backlogging and Weibull deterioration rate." European Journal of Operational Research, 192, 79-92.

[23] Wu, K.S. \& Ouyang, L.Y. (2000). “A Replenishment Policy for Deteriorating Items with Ramp Type Demand Rate.” Proc. Natl. Sci. Counc. ROC(A) ,24(4), 279-286.

[24] Wee, H. M.(1999). "Deteriorating inventory model with quantity discount, pricing and partial backordering." International Journal of Production Economics, 59, 511518.

[25] Wu, K., S.(2001). “An EOQ inventory model for items with Weibull distribution deterioration, ramp type demand rate and partial backlogging." Production Planning and Control, 12 (8), 787-793.

[26] Zhou, Y.W., Lau, H.S. and Yang S.L. (2004). "A finite horizon lot-sizing problem with time-varying deterministic demand and waiting-time-dependent partial backlogging." Int. J. Production Economics,91,109-119.

[27] Huang, Guobo, (1995). Modelling China's demand for international reserves. Applied Financial Economics, 5, pp. $357-366$.

[28] Yang, P.C. (2004). "Pricing strategy for deteriorating items using quantity discount when demand is price sensitive". European Journal of Operational Research, 157(2), 389-397.

[29] L. Davis, (1991). "The Handbook of Genetic Algorithms”, Van Nostrand Reinhold, New York .

[30] D.E. Goldberg,(1989). "Genetic Algorithms in Search, Optimization, and Machine Learning”, Addison-Wesley, Reading, MA .

[31] J.H. Holl and,(1975)." Adaptation in Natural and Artificial Systems", The University of Michigan Press, Ann Arbor, IL .

[32] Z. Michalewicz,1994," Genetic Algorithms + Data Structures = Evolution Programs". AI Series, Springer, New York. 